\title{
Probing the Nanoscale Features of Rhodobacter Sphaeroides: Insight Gained from Cryo- Focused Ion Beam and Cryo-Electron Tomography
}

\author{
Jade M. Noble $^{1}$, J. Lubieniecki ${ }^{2}$, J. Plitzko ${ }^{2}$, H. Engelhardt ${ }^{2}$, W. Baumeister ${ }^{2}$, and L. F. Kourkoutis ${ }^{3,4}$ \\ 1. School of Chemical and Biomolecular Engineering, Cornell University, Ithaca, New York, USA \\ 2. Dept. of Molecular Structural Biology, Max Planck Institute of Biochemistry, Martinsried, Germany. \\ 3. School of Applied and Engineering Physics, Cornell University, Ithaca, New York, USA. \\ 4. Kavli Institute at Cornell for Nanoscale Science, Ithaca, New York, USA.
}

Rhodobacter Spaheroides is a purple bacterium that contains several hundred chromatophore vesicles, which execute the bacteria's photosynthetic processes. The arrangement of vesicular proteins both within a single chromatophore and on its surface is crucial for efficient capture of excitation energy and conversion into usable chemical energy. Understanding the complete structure of an individual chromatophore and how neighboring chromatophores act in concert could further elucidate the photosynthetic processes in $R$. Sphaeroides bacteria.

While budding of chromatophores from the inner bacteria wall has been imaged by cryo-electron tomography of whole cells [1], the details of the bacteria's internal structure are resolved only on its comparatively thin peripheries. To visualize internal components at high spatial resolution, thinning of the cell is required while maintaining the structural integrity of the subcellular components. Here, we prepare vitrified TEM lamellae and wedges of $R$. Sphaeroides using cryo-focused ion beam (FIB) milling (Fig. 1a,b). Cryo-FIB milling uses a focused $\mathrm{Ga}^{+}$ion beam to create site-specific sections of frozen, hydrated biological specimens at cryogenic temperatures. A single TEM sample contains several bacteria to investigate, improving the throughput of the technique. The thickness of a typical cryo-FIB lamella of $R$. Sphaeroides was $\sim 150 \mathrm{~nm}$ (Fig. 1d), sufficiently thick to probe connectivity between neighboring, randomly oriented vesicles in all three dimensions.

Computational models of the surface arrangement of chromatophore proteins have been derived from a combination of experimental data using atomic force microscopy, electron microscopy [2-5]. Current models can be improved with a three-dimensional (3D) analysis of the vesicle without flattening its membrane. We used cryo-electron tomography (cryo-ET) to produce 3D visualizations of the spatial arrangement of the chromatophore vesicles and their connections in R. Sphaeroides. Cryo-FIB lamellae or wedge-shaped samples were introduced into the cryo-TEM for 3D image acquisition under low dose conditions. Tilt series of projection images of the bacteria were reconstructed into tomograms using computational, iterative algorithms (Fig. 1c,d, Fig. 2). Careful alignment of individual images in a tilt series before reconstruction is critical in reducing artifacts in the final tomogram. Gold particles are the traditional fiduciaries, but their high contrast can mask regions in the reconstruction. Here, $\mathrm{Ga}^{+}$ions embedded on the sample's surface during cryo-FIB milling provide the alignment fiduciaries (Fig. 1d).

High-resolution cryo-tomograms of the thinned bacteria reveal the bacterial membrane, several hundred chromatophores throughout the cell, and nanoscale intracellular components (Fig. 1c, 1d, 2a). Once believed to be isolated vesicles, chromatophores actually form a reticulum with neighboring vesicles (Fig. 2a,b). A crosssection of a reconstructed chromatophore (diameter: $58.7 \pm 2.3 \mathrm{~nm}$ ) reveals a double-membrane $\sim 4 \mathrm{~nm}$ thick embedded with high-intensity surface proteins that protrude into the vesicle's interior (Fig. 3a,b). The resolution obtained through sample thinning by cryo-FIB also exposed exclusively intervesicular proteins, which cannot be resolved through cryo-ET of full bacteria. The size of these proteins is $\sim 4 \mathrm{~nm}$, the approximate size of the shuttling cytochrome $c_{2}$ protein. Capitalizing on sample thinning techniques historically reserved for solid state materials allows us to probe the interior of $R$. Sphaeroides both on the micro- and the nanoscale, revealing subcellular structures inaccessible through traditional electron microscopy. 
[1] J.D. Tucker et al., Mol. Microbiol., 76 (2010), p. 833-847.

[2] Sener et al., Biophys. J., 104(2010), p. 15723-15728.

[3] S. Bahatyrova et al., Nature, 430 (2004), p.1058-1062.

[4] S. Scheuring et al., Biochem. Biophys. Acta, 8137(2014), p. 1263-1270.

[5] P. Qian et al., J. Mol. Biol., 349 (2005), p. 948-960.
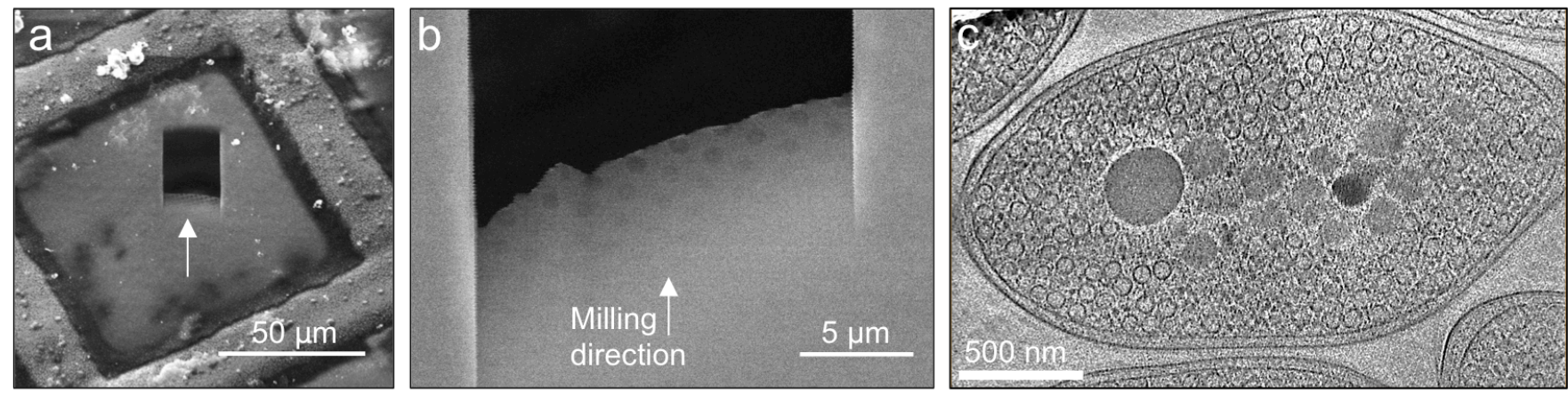

Fig. 1 (a) Cryo-FIB milling of wedge-shaped sample directly on the TEM grid. (b) SEM image of a cryo-FIB sample containing vitrified $R$. Sphaeroides. (c) Slice from a tomogram of an entire bacteria thinned by cryo-FIB, revealing cytoplasmic components. (d) Side-view of the tomogram. Ga clusters embedded in the sample surface served as reconstruction fiduciaries (arrow).
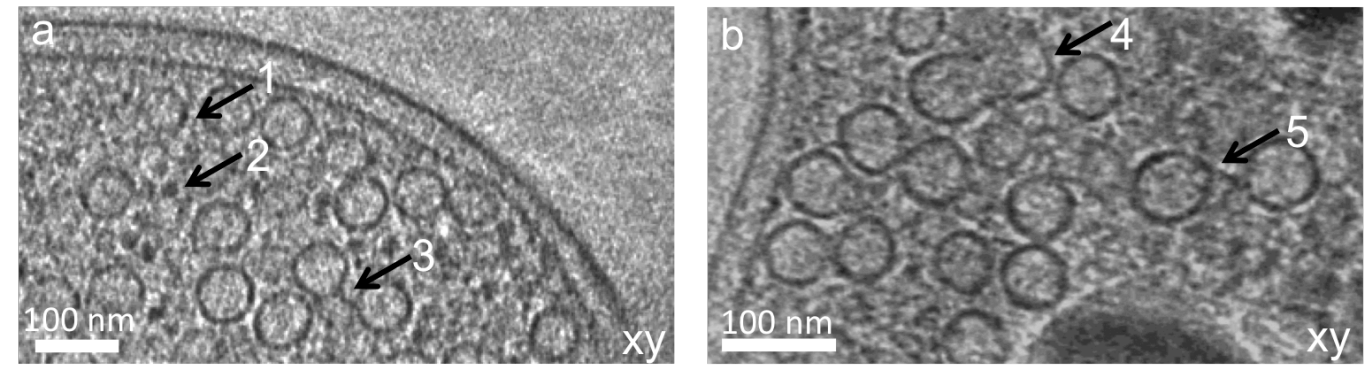

Fig. 2 (a) Orthoslices from reconstructed lamellae show nanoscale features such as ribosomes (1) and chromatophore surface proteins (2). (b) Cryo-FIB bacteria show that chromatophores often are fused (4) or form a reticulum with neighboring vesicles (a3, b5).
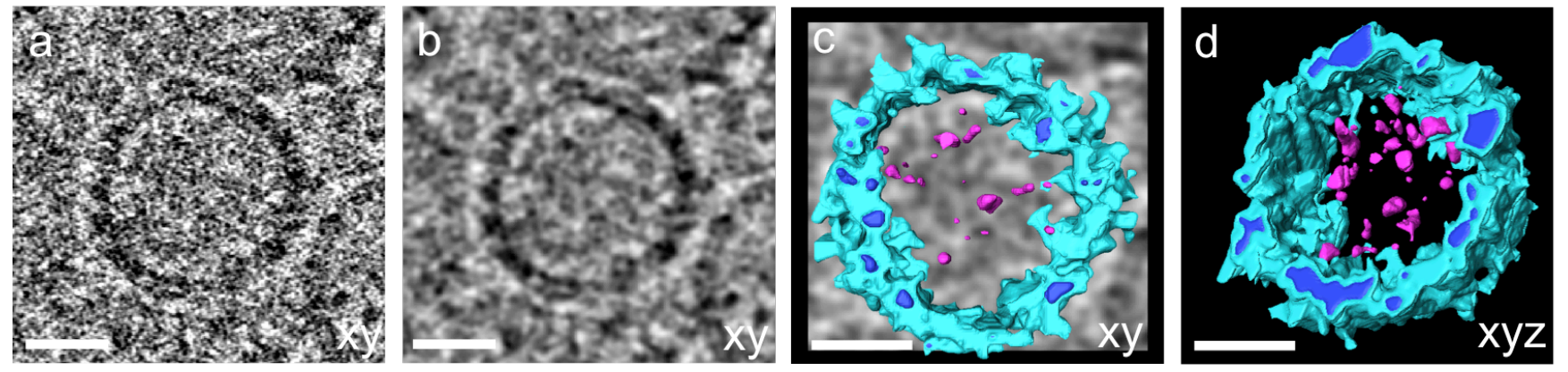

Fig. 3 (a) Tomographic slice of an individual chromatophore displays high-intensity features both on its surface and the interior. (b) For segmentation, the tomogram was denoised using anisotropic nonlinear diffusion and a Gaussian blur. (c,d) The tomogram is segmented so that the bacteria wall, surface proteins, and interior structures can be visualized in $3 \mathrm{D}$ along different axes. 